\title{
Comparação da sensibilidade ao contraste entre transplante lamelar anterior profundo e transplante penetrante para tratamento do ceratocone
}

\author{
Contrast sensitivity in deep anterior lamellar keratoplasty versus \\ penetrating keratoplasty
}

\author{
Carlos Anchieta Castro Cardoso da Silva ${ }^{1}$ \\ Ederson Schweitzer de Oliveira ${ }^{2}$ \\ Manoel Paulo Souza de Sena Júnior ${ }^{3}$ \\ Luciene Barbosa de Souza ${ }^{4}$
}

Trabalho realizado no Hospital Oftalmológico de Sorocaba / Banco de Olhos de Sorocaba - Sorocaba (SP) Brasil

Médico Oftalmologista, Estagiário dos Departamentos de Córnea e Cirurgia Refrativa do Hospital Oftalmológico de Sorocaba - Sorocaba (SP) - Brasil.

Médico Oftalmologista, Estagiário dos Departamentos de Córnea e Cirurgia Refrativa do Hospital Oftalmológico de Sorocaba - Sorocaba (SP) - Brasil.

Médico Residente do $3^{\circ}$ ano de Oftalmologia do Hospital Oftalmológico de Sorocaba - Sorocaba (SP) - Brasil.

${ }^{4}$ Doutora, Coordenadora do Setor de Córnea e Doenças

Externas do Hospital Oftalmológico de Sorocaba Sorocaba (SP) - Brasil.

Endereço para correspondência: Carlos Anchieta Castro Cardoso da Silva. Rua Nabeck Shiroma, 210 - Sorocaba (SP) CEP 18031-060

E-mail: carlosanchieta@oi.com.b

Recebido para publicação em 07.04.2007

Última versão recebida em 02.08 .2007

Aprovação em 03.08.2007

\begin{tabular}{|c|}
\hline RESUMO \\
\hline 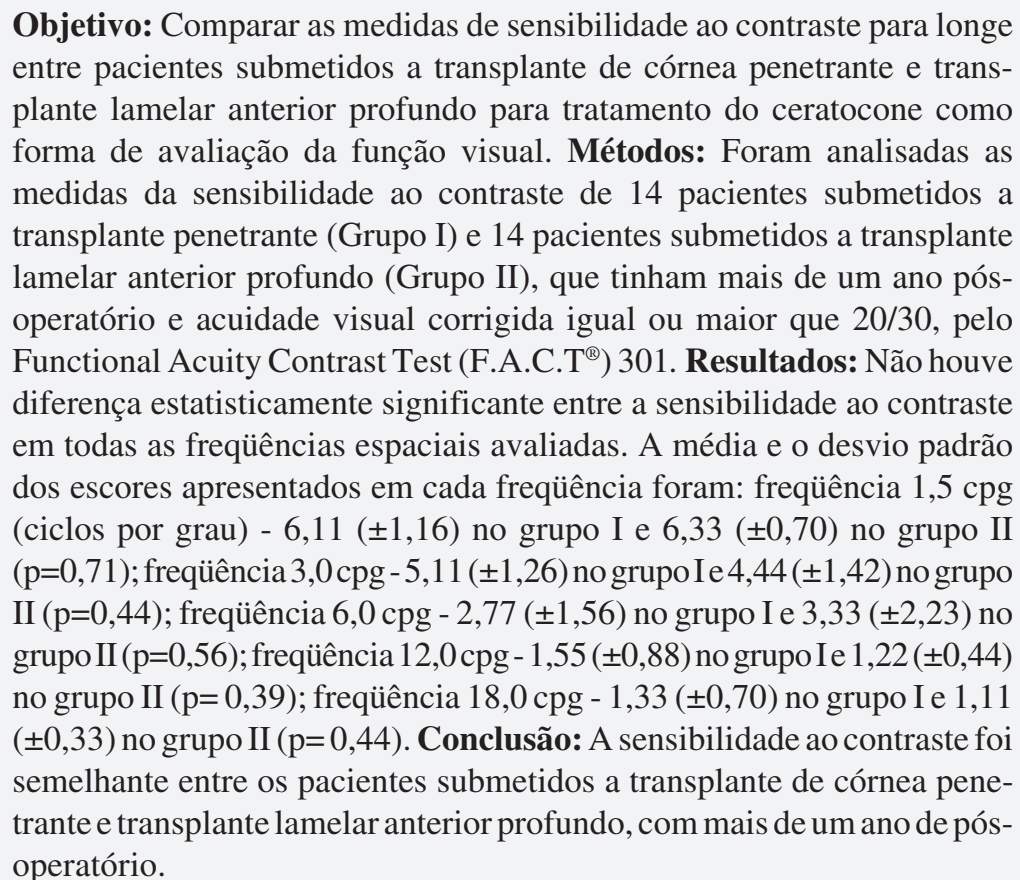 \\
\hline
\end{tabular}

Descritores: Córnea; Transplante de córnea; Doenças da córnea; Ceratocone/terapia; Sensibilidade de contraste

\section{INTRODUÇ̃̃OO}

O ceratocone é uma ectasia corneana bilateral com uma incidência de aproximadamente 1 por 2.000 na população geral ${ }^{(1)}$. Aparece tipicamente no final da adolescência e no adulto jovem e a idade média de apresentação é de 22,4 $\operatorname{anos}^{(2)}$. Apresenta-se como uma doença com tendência à progressão $^{(3)}$ e consiste na principal causa de indicação de transplante de córnea em adultos jovens ${ }^{(4-5)}$.

Recentes avanços em técnica cirúrgica promoveram uma troca de paradigma no tratamento cirúrgico das doenças da córnea. Transplante penetrante está sendo substituído agora por vários tipos de técnicas lamelares que apontam para substituir apenas o tecido alterado, enquanto se mantém o tecido saudável intacto ${ }^{(6)}$. 
O transplante lamelar anterior profundo é uma alternativa segura para o tratamento cirúrgico do ceratocone apresentando resultados de acuidade visual semelhantes ao transplante penetrante e sem risco de rejeição endotelial ${ }^{(7-9)}$.

A medida de acuidade visual de Snellen é utilizada há muito tempo como o teste de função visual primário de sucesso cirúrgico. Porém, alguns pacientes informam limitações da visão apesar de um enxerto transparente e de boa acuidade visual. Durante as últimas duas décadas tem-se demonstrado evidências de que existem outros aspectos de função visual além da acuidade em pacientes que têm opacidades de meios e irregularidade de córnea ${ }^{(10-13)}$.

Um destes aspectos de função visual consiste na sensibilidade ao contraste, que tem sido um indicador de qualidade visual amplamente apreciado $^{(14)}$.

Este estudo pretende avaliar se existe diferença na qualidade da função visual através da sensibilidade ao contraste para longe entre pacientes submetidos a transplante de córnea penetrante e transplante lamelar anterior profundo para tratamento do ceratocone.

\section{MÉTODOS}

Consentimento informado foi obtido de todos os pacientes após ter sido explicada a natureza do estudo.

Critérios de inclusão foram acuidade visual de Snellen corrigida igual ou maior a 20/30 em pacientes de idade maior ou igual a 14 anos submetidos ao transplante de córnea por diagnóstico de ceratocone, com um curso pós-operatório sem complicações de pelo menos 12 meses.

O diagnóstico de ceratocone foi baseado no exame sob lâmpada de fenda, topografia computadorizada de córnea e refração.

Critérios de exclusão foram acuidade visual Snellen corrigida inferior a 20/30, histórico de cirurgia ocular prévia e complicações intra ou pós-operatórias.

Os pacientes foram divididos em dois grupos:

- Grupo I - Pacientes submetidos a transplante penetrante.

- Grupo II - Pacientes submetidos a transplante lamelar anterior profundo.

Todas as córneas doadoras foram obtidas do Banco de Olhos de Sorocaba e estavam conservadas em meio de cultura Optisol $^{\circledR}$.

Os transplantes lamelares anteriores profundos foram realizados através da técnica de dissecção profunda com ar mantendo-se a membrana de Descemet e o endotélio da córnea doadora intactos.

Foram coletados dados da acuidade visual de Snellen (AV) e teste de sensibilidade ao contraste (SC) de 14 olhos de 14 pacientes em cada grupo de estudo. Os dados foram coletados em uma única sessão. Todos os pacientes fizeram uso da melhor correção visual.

O Teste de Sensibilidade ao Contraste foi realizado com o Functional Acuity Contrast Test - F.A.C.T ${ }^{\circledR} 301$ (Stereo Op- tical, Chicago, IL). Foi realizado a uma distância de 3 metros (10 pés) com iluminação uniforme entre $68-240 \mathrm{~cd} / \mathrm{m}^{2}$. O teste é composto por uma lâmina com apresentação de gradeados que avaliam a sensibilidade ao contraste em cinco frequiências espaciais: $1,5 \mathrm{cpg}$ (ciclos por grau), $3,0 \mathrm{cpg}, 6,0 \mathrm{cpg}$, $12,0 \mathrm{cpg}$ e $18,0 \mathrm{cpg}$. O resultado pode ser avaliado em cada frequiência espacial separadamente e, também globalmente, utilizando-se um valor único para o exame através de um equivalente à tabela de Snellen.

Foram comparadas as médias de cada frequiência espacial e os valores do equivalente funcional de Snellen.

Foi utilizado o teste $t$ de Student para a verificação da significância da diferença entre os valores médios dos dois grupos. Foi fixado o nível de significância de 5\% para todas as análises estatísticas $(\mathrm{p}<0,05)$.

Outras informações coletadas incluíram dados demográficos.

\section{RESULTADOS}

A idade dos pacientes variou de 21 a 34 anos, com média de 26,7 $\pm 5,5$ no Grupo I e de 18 a 49 anos, com média de 29,8 $\pm 12,9$ no Grupo II (Tabela 1 ).

No Grupo I (transplante penetrante) $7(50 \%)$ pacientes pertenciam ao sexo feminino e 7 (50\%) pacientes pertenciam ao sexo masculino. No Grupo II 7 (50\%) pacientes pertenciam ao sexo masculino e $7(50 \%)$ pacientes pertenciam ao sexo feminino (Tabela 1).

A acuidade visual com melhor correção no Grupo I foi 20/30 em 7 (50\%) pacientes, 20/25 em 7 (50\%) pacientes. No Grupo II foi 20/30 em 7 (50\%) pacientes e 20/25 em 7 (50\%) pacientes. As médias de acuidade visual em valores decimais no Grupo I e Grupo II foram, respectivamente, $0,72( \pm 0,07) \mathrm{e}$ $0,72( \pm 0,07)$ (Tabela 2).

Não houve diferença estatisticamente significante entre a sensibilidade ao contraste para longe em todas as frequiências espaciais avaliadas nos dois grupos. A média e o desvio padrão dos escores apresentados em cada freqüência espacial foram: frequiência $1,5 \mathrm{cpg}$ (ciclos por grau) - $6,11( \pm 1,16)$ no

\begin{tabular}{|lcc|}
\hline \multicolumn{3}{c}{ Tabela 1. Perfil dos pacientes } \\
Idade média & Grupo I & Grupo II \\
Sexo & $26,7( \pm 5,5)$ & $29,8( \pm 12,9)$ \\
& $50,0 \%$ masculino & $50,0 \%$ feminino \\
& $50,0 \%$ masculino & $50,0 \%$ feminino \\
\hline
\end{tabular}

\begin{tabular}{|lcc|}
\hline \multicolumn{3}{|c|}{ Tabela 2. Acuidade visual corrigida } \\
AV & Grupo I & Grupo II \\
$20 / 30$ & 7 pacientes $(50,0 \%)$ & 7 pacientes $(50,0 \%)$ \\
$20 / 25$ & 7 pacientes $(50,0 \%)$ & 7 pacientes $(50,0 \%)$ \\
$A V=$ acuidade visual & \\
\hline
\end{tabular}


grupo I e 6,33 $( \pm 0,70)$ no grupo II ( $\mathrm{p}=0,71)$; freqüência $3,0 \mathrm{cpg}$ - 5,11 $( \pm 1,26)$ no grupo I e $4,44( \pm 1,42)$ no grupo II $(\mathrm{p}=0,44)$; frequiência $6,0 \mathrm{cpg}-2,77( \pm 1,56)$ no grupo I e $3,33( \pm 2,23)$ no grupo II ( $\mathrm{p}=0,56)$; frequiência $12,0 \mathrm{cpg}-1,55( \pm 0,88)$ no grupo I e $1,22( \pm 0,44)$ no grupo II $(\mathrm{p}=0,39)$; freqüência $18,0 \mathrm{cpg}-1,33$ $( \pm 0,70)$ no grupo I e $1,11( \pm 0,33)$ no grupo II $(\mathrm{p}=0,44)$ (Tabela 3 e Gráfico 1).

Usando-se o equivalente funcional de Snellen, em valores decimais, o Grupo I apresentou a média de $0,28( \pm 0,12)$ e o Grupo II apresentou a média de $0,27( \pm 0,12)$. $(\mathrm{p}=0,87)$. Não houve diferença estatisticamente significante (Tabela 4).

\section{DISCUSSÃO}

Em vários estudos os autores têm concluído que o transplante lamelar anterior profundo apresenta bom resultado visual para o tratamento do ceratocone, não apresentando falência primária ou rejeição endotelial ${ }^{(7-9,15)}$.

Alguns trabalhos concluíram que a acuidade visual corrigida apresentou resultados semelhantes entre pacientes submetidos a transplante penetrante e transplante lamelar anterior profundo por diagnóstico de ceratocone ${ }^{(8,16)}$.

\begin{tabular}{|c|c|c|c|c|c|}
\hline \multirow{2}{*}{$\begin{array}{l}\text { Freqüência } \\
\text { espacial }\end{array}$} & \multicolumn{2}{|c|}{ Grupo I } & \multicolumn{2}{|c|}{ Grupo II } & \multirow{2}{*}{ Significância } \\
\hline & $\begin{array}{l}\text { Escore } \\
\text { médio }\end{array}$ & $\begin{array}{l}\text { Desvio } \\
\text { padrão }\end{array}$ & $\begin{array}{l}\text { Escore } \\
\text { médio }\end{array}$ & $\begin{array}{l}\text { Desvio } \\
\text { padrão }\end{array}$ & \\
\hline $1,5 \mathrm{cpg}$ & 6,1 & $\pm 1,16$ & 6,3 & $\pm 0,70$ & $p=0,71$ \\
\hline $3,0 \mathrm{cpg}$ & 5,1 & $\pm 1,26$ & 4,4 & $\pm 1,42$ & $p=0,44$ \\
\hline $6,0 \mathrm{cpg}$ & 2,7 & $\pm 1,56$ & 3,3 & $\pm 2,23$ & $p=0,56$ \\
\hline $12,0 \mathrm{cpg}$ & 1,5 & $\pm 0,88$ & 1,2 & $\pm 0,44$ & $p=0,39$ \\
\hline $18,0 \mathrm{cpg}$ & 1,3 & $\pm 0,70$ & 1,1 & $\pm 0,33$ & $p=0,44$ \\
\hline
\end{tabular}

\begin{tabular}{|c|c|c|}
\hline Grupo I & Grupo II & Significância \\
\hline Média Desvio padrão & Média Desvio padrão & \\
\hline $0,28 \quad \pm 0,12$ & $0,27 \quad \pm 0,12$ & $p=0,87$ \\
\hline (Significância p<0,05) & & \\
\hline
\end{tabular}

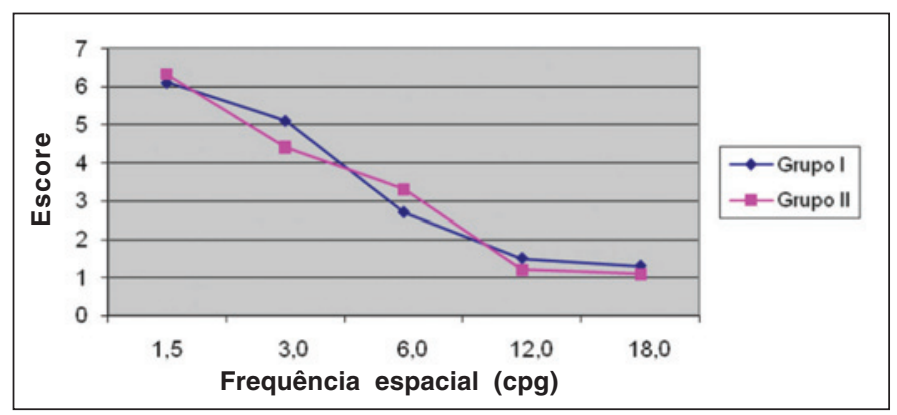

Gráfico 1 - Resultados da sensibilidade ao contraste
Vários estudos demonstram a utilidade do teste de sensibilidade ao contraste para aplicação como acompanhamento da progressão do ceratocone e como avaliação de sucesso para transplantes ${ }^{(16-19)}$.

Em recente estudo os autores demonstraram que não houve diferença entre a sensibilidade ao contraste entre pacientes submetidos a transplante penetrante e transplante lamelar anterior profundo por ceratocone. Concluíram, também, que a acuidade visual pós-transplante lamelar profundo é dependente da quantidade de estroma residual no leitor receptor, com melhor acuidade nos casos onde há menor quantidade residual $^{(19)}$.

No presente estudo a sensibilidade ao contraste para longe mostrou-se estatisticamente semelhante em todas as frequiências espaciais analisadas ( $1,5 \mathrm{cpg} ; 3,0 \mathrm{cpg} ; 6,0 \mathrm{cpg} ; 12,0 \mathrm{cpg}$ e $18,0 \mathrm{cpg}$ ) entre o grupo submetido a transplante penetrante e o grupo submetido a transplante lamelar anterior profundo.

\section{CONCLUSÃO}

O transplante lamelar anterior profundo apresentou resultados semelhantes de sensibilidade ao contraste para longe comparado ao transplante penetrante para tratamento do ceratocone em pacientes com mais de um ano de pós-operatório.

\section{ABSTRACT}

Purpose: To compare the measurement of contrast sensitivity at a distance in patients submitted to penetrating keratoplasty versus patients submitted to deep anterior lamellar keratoplasty for keratoconus treatment. Methods: Contrast sensitivity of fourteen subjects submitted to penetrating keratoplasty and fourteen subjects submitted to deep anterior lamellar keratoplasty have been analyzed through the Functional Acuity Contrast Test (F.A.C.T ${ }^{\circledR}$ ) 301. Results: There was no statistically significant difference between the measurements of either penetrating keratoplasty or deep anterior lamellar keratoplasty. Conclusion: Contrast sensitivity was similar among the subjects submitted to penetrating keratoplasty and to deep anterior lamellar keratoplasty.

Keywords: Cornea; Corneal transplantation; Corneal diseases; Keratoconus/therapy; Contrast sensitivity

\section{REFERÊNCIAS}

1. Rabinowitz YS. Keratoconus. Surv Ophthalmol. 1998;42(4):297-319.

2. Tuft SJ, Moodaley LC, Gregory WM, Davison CR, Buckley RJ. Prognostic factors for the progression of keratoconus. Ophthalmology. 1994;101(3):439-47.

3. Krachmer JH, Feder RS, Belin MW. Keratoconus and related noninflammatory corneal thinning disorders. Surv Ophthalmol. 1984;28(4):293-322.

4. Calix Netto MJ, Giustina ED, Ramos GZ, Peccini RF, Sobrinho M, de Souza LB. Principais indicações de transplante penetrante de córnea em um serviço de referência no interior de São Paulo (Sorocaba (SP) - Brasil). Arq Bras Oftalmol. 2006;69(5):661-4. 
5. Vail A, Gore SM, Bradley BA, Easty DL, Rogers CA. Corneal graft survival and visual outcome. A multicenter Study. Corneal Transplant Follow-up Study Collaborators. Ophthalmology 1994;101(1):120-7.

6. Shimmura S, Tsubota K. Deep anterior lamellar keratoplasty. Curr Opin Ophthalmol. 2006;17(4):349-55. Review.

7. Watson SL, Ramsay A, Dart JK, Bunce C, Craig E. Comparison of deep lamellar keratoplasty and penetrating keratoplasty in patients with keratoconus. Ophthalmology. 2004;111(9):1676-82.

8. Vabres B, Bosnjakowski M, Bekri L, Weber M, Pechereau A. [Deep lamellar keratoplasty versus penetrating keratoplasty for keratoconus]. J Fr Ophtalmol. 2006;29(4):361-71. French.

9. Funnell CL, Ball J, Noble BA. Comparative cohort study of the outcomes of deep lamellar keratoplasty and penetrating keratoplasty for keratoconus. Eye. 2006;20(5):527-32.

10. Rubin GS, Adamsons IA, Stark WJ. Comparison of acuity, contrast sensitivity, and disability glare before and after cataract surgery. Arch Ophthalmol. 1993;111(1):56-61.

11. Bodis-Wollner I. Detection of visual defects using the contrast sensitivity function. Int Ophthalmol Clin. 1980;20(1):135-53.

12. Elliott DB, Hurst MA, Weatherill J. Comparing clinical tests of visual function in cataract with the patient's perceived visual disability. Eye. 1990;4(Pt 5):712-7.

13. Mannis MJ, Zadnik K, Johnson CA. The effect of penetrating keratoplasty on contrast sensitivity in keratoconus. Arch Ophthalmol. 1984;102(10):1513-6.

14. McLeod SD. Beyond Snellen acuity: the assessment of visual function after refractive surgery. Arch Ophthalmol. 2001;119(9):1371-3.

15. Fournié P, Coullet J, Moalic S, Malecaze F, Chapotot E, Arné JL. [Deep anterior lamellar keratoplasty in the surgical treatment of keratoconus. A 1year follow-up]. J Fr Ophtalmol. 2006;29(6):602-13. French.

16. Brahma A, Ennis F, Harper R, Ridgway A, Tullo A. Visual function after penetrating keratoplasty for keratoconus: a prospective longitudinal evaluation. Br J Ophthalmol. 2000;84(1):60-6.

17. Pesudovs K, Schoneveld P, Seto RJ, Coster DJ. Contrast and glare testing in keratoconus and after penetrating keratoplasty. Br J Ophthalmol. 2004;88(5): 653-7.

18. Zadnik K, Mannis MJ, Johnson CA. An analysis of contrast sensitivity in identical twins with keratoconus. Cornea. 1984;3(2):99-103.

19. Ardjomand N, Hau S, McAlister JC, Bunce C, Galaretta D, Tuft SJ, Larkin DF. Quality of vision and graft thickness in deep anterior lamellar and penetrating corneal allografts. Am J Ophthalmol. 2007;143(2):228-35.

\title{
Congresso comemorativo
dos 65 anos do Hospital de Olhos
Sadalla Amin Ghanem
em parceria com a
Sociedade Catarinense de Oftalmologia
}

\section{Hotel Bourbon \\ Joinville - SC}

14 e 15 de março de 2008

\author{
INFORMAÇÕES \\ Agência Criacom \\ Tel.: (47) 3028-5850 \\ E-mail: marcela@criacomcomunica.com.br \\ home page: www.sadalla.com.br/congresso
}

